\title{
Variational Principles in Quantum Monte Carlo: The Troubled Story of Variance Minimization
}

\author{
Alice Cuzzocrea, Anthony Scemama,* Wim J. Briels,* Saverio Moroni,* and Claudia Filippi* \\ Cite This: J. Chem. Theory Comput. 2020, 16, 4203-4212 \\ Read Online
}

ABSTRACT: We investigate the use of different variational principles in quantum Monte Carlo, namely, energy and variance minimization, prompted by the interest in the robust and accurate estimation of electronic excited states. For two prototypical, challenging molecules, we readily reach the accuracy of the best available reference excitation energies using energy minimization in a state-specific or state-average fashion for states of different or equal symmetry, respectively. On the other hand, in variance minimization, where the use of suitable functionals is expected to target specific states regardless of the symmetry, we encounter severe problems for a variety of wave functions: as the variance converges, the energy drifts away from that of

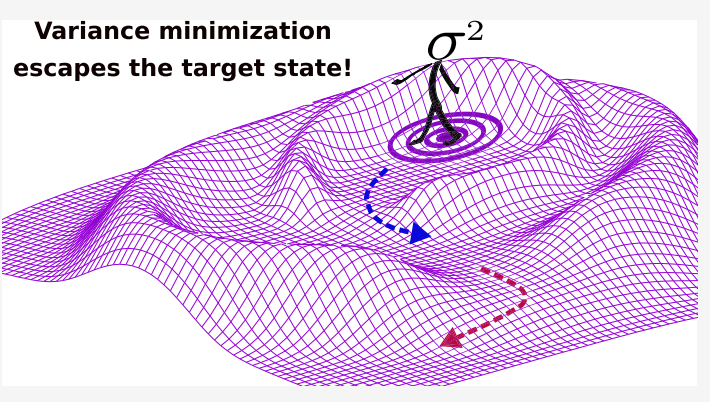
the selected state. This unexpected behavior is sometimes observed even when the target is the ground state and generally prevents the robust estimation of total and excitation energies. We analyze this problem using a very simple wave function and infer that the optimization finds little or no barrier to escape from a local minimum or local plateau, eventually converging to a lower-variance state instead of the target state. For the increasingly complex systems becoming in reach of quantum Monte Carlo simulations, variance minimization with current functionals appears to be an impractical route.

\section{INTRODUCTION}

Light-induced processes are at the heart of a variety of phenomena and applications which range from harnessing the response to light of biological systems to improving the technologies for renewable energies. The contribution of electronic structure theory in this field hinges on its ability to efficiently and accurately compute excited-state properties. In this context, the use of quantum Monte Carlo (QMC) methods is relatively recent and quite promising: ${ }^{1-9}$ QMC approaches provide an accurate (stochastic) solution of the Schrödinger equation and benefit from a favorable scaling with system size and great ease of parallelization. ${ }^{10-12}$ Importantly, recent methodological advancements ${ }^{13-16}$ have enabled the fast calculation of energy derivatives and the optimization of many thousands of parameters for the internally consistent computation of QMC wave functions and geometries in the ground and excited states., 17

Here, we investigate the use of two different variational principles for ground and excited states in QMC, namely, variance and energy minimization, to assess whether they allow us to fully capitalize on the increased power of minimization algorithms and availability of accurate wave functions. Variance minimization techniques ${ }^{18-22}$ have been extensively employed in QMC for the last 30 years, but their potential for the computation of excited states has only recently been revisited and exploited to compute vertical excitation energies of various small molecules. ${ }^{23,24}$ Different functionals for the optimization of the variance ${ }^{19,22,23}$ have also been put forward with the common attractive feature of the built-in possibility to target a specific state and avoid, in principle, the complications encountered in energy minimization where, without constraints, one would generally collapse to lower-energy states.

For our study, we select two molecules, a small cyanine dye and a retinal model, because of the difficulties they pose in the computation of the lowest vertical excitation energy ${ }^{4,25-28}$ and the different requirements in the procedure adopted in energy minimization: while the ground and excited states of the cyanine belong to different symmetries and can therefore be treated in a state-specific manner, this is not the case for the retinal model, where energy minimization must be performed in a state-average fashion. For both molecules and therefore regardless of the nature of the optimization, we find that energy minimization leads to the stable and fast convergence of the total energies of the states of interest. Furthermore, with the use of compact and balanced energy-minimized wave functions constructed through a selected configuration interaction (CI) approach, we recover vertical excitation energies which are already at the variational Monte Carlo

Received: February 13, 2020

Published: May 17, 2020 
(VMC) level within chemical accuracy (about $0.04 \mathrm{eV}$ ) of the reference coupled cluster or extrapolated CI values. On the other hand, for both molecules and for nearly all wave functions investigated, the optimization of all parameters in variance minimization is problematic because it results in the apparent loss of the state of interest over sufficiently long optimization runs, precluding the estimate of the excitation energy. This occurs for the different functionals originally proposed to stabilize the optimization and, surprisingly, in some cases, also when targeting the ground state. This finding is unexpected, especially considering that variance minimization has been the method of choice in QMC for decades and is still routinely used, albeit for simpler systems and/or for wave functions with a small number of parameters, often limited to the Jastrow factor.

To understand these newly found issues, we examine how variance minimization behaves when optimizing the linear coefficients of a very small CI expansion: working in the linear subspace spanned by a few approximate eigenvectors, we discover that the optimization of the $\mathrm{CI}$ parameters in variance minimization does not converge to the target eigenstate but to a different one. In this specific example, during the minimization, the system slowly reaches the eigenstate corresponding to the absolute minimum of the variance, no matter what the starting state is. We verify that a similar pattern explains the unexpected behavior observed for more complicated wave functions.

It is well known that the variance reaches its minimum value of zero for every exact eigenstate. ${ }^{19}$ This is the very basis of variance minimization. Whether the variance maintains a minimum when any particular eigenstate is described by a given approximate wave function is a question that can only be assessed empirically on a case-by-case basis. Our calculations identify missing minima in several instances of current interest for QMC simulations. Systematic improvement of the wave function to recover the zero-variance property of the exact eigenstates would be possible, in principle, but impractically demanding.

Our findings pose severe limitations on the application of variance minimization for the increasingly complex systems that are becoming accessible to QMC simulations.

In Section 2, we recap the equations used for energy and variance optimization, discuss the procedure employed for the state-average case, and introduce the ingredients for a stable version of the Newton method in variance minimization. In Section 3, we summarize the computational details, and in Section 4, we present the accurate vertical excitation energies obtained in energy minimization and the difficulties encountered in variance minimization for both molecules. We elucidate these findings and conclude in Section 5.

\section{METHODS}

We briefly introduce below the variance and energy minimization approaches used to optimize the wave functions in variational Monte Carlo. While we employ variance minimization as a state-specific approach to target a given state, we must distinguish between a state-specific and a stateaverage route for energy optimization when the excited state of interest is of different or equal symmetry, respectively, than other lower-lying states.

2.1. Wave Function Form. The wave functions employed in this work are of the Jastrow-Slater type, namely, the product of a determinantal expansion and a Jastrow correlation function, $\mathcal{J}$, as

$$
\Psi=\mathcal{J} \sum_{i=1}^{N_{\mathrm{det}}} c_{i} D_{i}
$$

where the determinants are expressed on single-particle orbitals and the Jastrow factor includes an explicit dependence on the electron-electron distances. Here, the Jastrow factor is chosen to include electron-electron and electron-nucleus correlation terms. ${ }^{29}$ For the determinantal component, we select the relevant determinants according to different recipes: (i) very simple ansatzes such as Hartree-Fock (HF) or a CI singles (CIS) expansion recently put forward as a computationally cheap and sufficiently accurate wave function for excited states in $\mathrm{QMC}^{8,30}$ (ii) complete-active-space (CAS) expansions where small sets of important active orbitals are manually identified; and (iii) CI perturbatively selected iteratively (CIPSI) expansions generated to yield automatically balanced multiple states for a fast convergence of the QMC excitation energy with the number of determinants. All expansions are expressed in terms of spin-adapted configuration state functions (CSFs) to reduce the number of variational parameters.

2.2. Energy Minimization. For state-specific optimization in energy minimization, we employ the stochastic reconfiguration (SR) method ${ }^{14,31}$ in a low-memory conjugate-gradient implementation. ${ }^{14}$ Given a starting wave function $\Psi$ depending on a set of parameters $p$, we denote the derivatives of $\Psi$ with respect to the parameter $p_{i}$ as $\Psi_{i}=\partial_{\mathrm{i}} \Psi$. At every step of SR optimization, the parameter variations, $\Delta p$, are computed according to the equation

$$
\bar{S} \Delta p=-\tau g
$$

where $\tau$ is a positive quantity chosen small enough to guarantee the convergence. The vector $g$ is the gradient of the energy with components

$$
\begin{aligned}
g_{i}=\frac{\partial E}{\partial p_{i}} & =2\left[\frac{\left\langle\Psi_{i}|\hat{\mathcal{H}}| \Psi\right\rangle}{\langle\Psi \mid \Psi\rangle}-E \frac{\left\langle\Psi \mid \Psi_{i}\right\rangle}{\langle\Psi \mid \Psi\rangle}\right] \\
& =2\left[\left\langle\frac{\Psi_{i}}{\Psi} E_{\mathrm{L}}\right\rangle-\left\langle E_{\mathrm{L}}\right\rangle\left\langle\frac{\Psi_{i}}{\Psi}\right\rangle\right]
\end{aligned}
$$

where $E_{\mathrm{L}}=\hat{H} \Psi / \Psi$ is the so-called local energy and $\langle$.$\rangle denotes$ the Monte Carlo average of the quantity in brackets over the electron configurations sampled from $\Psi^{2} /\langle\Psi \mid \Psi\rangle$. The matrix $\bar{S}$ has components

$$
\begin{aligned}
\bar{S}_{i j} & =\frac{\left\langle\Psi_{i} \mid \Psi_{j}\right\rangle}{\langle\Psi \mid \Psi\rangle}-\frac{\left\langle\Psi \mid \Psi_{i}\right\rangle}{\langle\Psi \mid \Psi\rangle} \frac{\left\langle\Psi \mid \Psi_{j}\right\rangle}{\langle\Psi \mid \Psi\rangle} \\
& =\left\langle\frac{\Psi_{i}}{\Psi} \frac{\Psi_{j}}{\Psi}\right\rangle-\left\langle\frac{\Psi_{i}}{\Psi}\right\rangle\left\langle\frac{\Psi_{j}}{\Psi}\right\rangle \equiv\left\langle\frac{\bar{\Psi}_{i}}{\Psi} \frac{\bar{\Psi}_{j}}{\Psi}\right\rangle
\end{aligned}
$$

which are expressed in the last equality as the overlap matrix in the semi-orthogonal basis, $\bar{\Psi}_{i}=\Psi_{i}-\left[\left\langle\Psi \mid \Psi_{i}\right\rangle /\langle\Psi \mid \Psi\rangle\right] \Psi$.

When the state of interest is energetically not the lowest in its symmetry class, we start from a set of wave functions for the multiple states which share the same Jastrow factor and orbitals but are characterized by different linear CI coefficients as 


$$
\Psi^{I}=\mathcal{J} \sum_{i=1}^{N_{\mathrm{det}}} c_{i}^{I} D_{i}
$$

where the superscript $I$ indicates a particular state. To obtain a balanced description of the states of interest, we optimize the nonlinear parameters of the orbitals and the Jastrow factor by minimizing the state-average energy ${ }^{1}$

$$
E^{\mathrm{SA}}=\sum_{I} w_{I} \frac{\left\langle\Psi^{I}|\hat{\mathcal{H}}| \Psi^{I}\right\rangle}{\left\langle\Psi^{I} \mid \Psi^{I}\right\rangle}
$$

where the weights $w_{I}$ are kept fixed and $\sum_{I} w_{I}=1$. To this aim, we follow the SR scheme (eq 2) and use the gradient of the state-average energy

$$
g_{i}^{\mathrm{SA}}=\sum_{I} w_{I} g_{i}^{I}
$$

where $g_{i}^{I}$ is the derivative with respect to the parameter $p_{i}$ of the energy of state $I$, which is computed from the wave function $\Psi^{I}$ and its derivatives, as in eq 3. Moreover, in analogy to singlestate optimization, we introduce a weighted-average overlap matrix defined as

$$
\bar{S}_{i j}^{\mathrm{SA}}=\sum_{I} w_{I} \bar{S}_{i j}^{I}
$$

where the overlap matrix for each state is computed from the corresponding wave function, as in eq 4 . We stress that although the state-average SR procedure is defined simply by analogy with the single-state case, it employs the correct gradients of the SA energy $\left(g^{\mathrm{SA}}\right)$ and, therefore, at convergence, it leads to the minimization of the state-average energy.

We alternate a number of optimization steps of the nonlinear parameters with the optimization of the linear coefficients $c_{i}^{I}$, whose optimal values are the solution of the generalized eigenvalue equations

$$
H^{\mathrm{CI}}{ }^{I}=E_{I} S^{\mathrm{CI}} c^{I}
$$

where the Hamiltonian and overlap matrix elements are defined on the basis of the functions $\left\{\mathcal{J} D_{i}\right\}$ and estimated through Monte Carlo sampling. After diagonalization of eq 9, orthogonality between the individual states is automatically enforced. To solve the eigenvalue equation with a memory efficient algorithm, we use the Davidson diagonalization scheme in which the lowest energy eigenvalues are computed without the explicit construction of the entire Hamiltonian and overlap matrices. ${ }^{14} \mathrm{~A}$ similar procedure has recently been followed in ref 32.

2.3. Variance Minimization. To perform variance minimization, we can directly minimize the variance of the state of interest

$$
\sigma^{2}=\frac{\left\langle\Psi\left|(\hat{\mathcal{H}}-E)^{2}\right| \Psi\right\rangle}{\langle\Psi \mid \Psi\rangle}
$$

or follow a somewhat more stable optimization procedure by minimizing the expression

$$
\sigma_{\omega}{ }^{2}=\frac{\left\langle\Psi\left|(\hat{\mathcal{H}}-\omega)^{2}\right| \Psi\right\rangle}{\langle\Psi \mid \Psi\rangle}
$$

where the energy $\omega$ is fixed during the optimization step and then appropriately modified to follow the current value of the energy, as originally proposed in ref 19 . Recently, the functional $\Omega$ has been put forward

$$
\Omega=\frac{\langle\Psi|(\omega-\hat{\mathcal{H}})| \Psi\rangle}{\left\langle\Psi\left|(\omega-\hat{\mathcal{H}})^{2}\right| \Psi\right\rangle}
$$

whose minimization is equivalent to variance minimization if $\omega$ is eventually updated to the running value of $E-\sigma .^{23}$

Because of its simplicity, here, we choose the functional $\sigma_{\omega}{ }^{2}$ but also compare the convergence behavior obtained with the functional $\Omega$. To this aim, we use the Newton optimization method, as in ref 22 , and update the parameters as

$$
\Delta p=-\tau h^{-1} g
$$

where $g$ is, here, the gradient of $\sigma_{\omega}{ }^{2}$ and $h$ is its Hessian matrix, and the parameter $\tau$ is introduced to damp the size of the variations.

The components of the gradient are given by

$$
\begin{aligned}
g_{i}= & 2\left[\left\langle\frac{\hat{\mathcal{H}} \Psi_{i}}{\Psi} E_{\mathrm{L}}\right\rangle-\left\langle\frac{\Psi_{i}}{\Psi}\right\rangle\left\langle E_{\mathrm{L}}{ }^{2}\right\rangle\right. \\
& \left.-\omega\left(\left\langle\frac{\Psi_{i}}{\Psi} E_{\mathrm{L}}\right\rangle+\left\langle\frac{\hat{H} \Psi_{i}}{\Psi}\right\rangle-2\left\langle\frac{\Psi_{i}}{\Psi}\right\rangle\left\langle E_{\mathrm{L}}\right\rangle\right)\right]
\end{aligned}
$$

and we discuss other possible equivalent expressions and their relative fluctuations in Section S1. The Hessian matrix elements require the second derivatives of the wave function and, to avoid their computation, we follow the same approximation strategy of the Levenberg-Marquardt algorithm ${ }^{33}$ and manipulate the expression of the variance in a somewhat different way than that proposed in refs $20,22,34$ to obtain the approximate expression of the Hessian matrix

$$
\begin{aligned}
h_{i j}= & \left\langle\left[\partial_{i} E_{\mathrm{L}}+\left(E_{\mathrm{L}}-\omega\right)\left(\frac{\Psi_{i}}{\Psi}-\left\langle\frac{\Psi_{i}}{\Psi}\right\rangle\right)\right]\right. \\
& \left.\times\left[\partial_{j} E_{\mathrm{L}}+\left(E_{\mathrm{L}}-\omega\right)\left(\frac{\Psi_{j}}{\Psi}-\left\langle\frac{\Psi_{j}}{\Psi}\right\rangle\right)\right]\right\rangle
\end{aligned}
$$

Details of the derivation and alternative expressions for the Hessian are given in Section S1.

We use the Newton method and the Hessian $h$ (eq 15) when optimizing both $\sigma_{\omega}{ }^{2}$ and the $\Omega$ functional in combination with the corresponding gradient. Furthermore, we follow ref 23 in keeping $\omega$ fixed to an appropriate guess energy for an initial number of minimization steps, upgrading it linearly to the running energy (or $E-\sigma$ in the case of $\Omega$ ) over some intermediate iteration steps, and then setting it equal to the current energy estimate for the rest of the run.

\section{COMPUTATIONAL DETAILS}

All QMC calculations are carried out with the program package CHAMP. ${ }^{35}$ We employ scalar-relativistic energyconsistent HF pseudopotentials and the correlation-consistent Gaussian basis sets specifically constructed for these pseudopotentials. $^{36,37}$ Unless otherwise specified, we use a double- $\zeta$ basis set minimally augmented with $s$ and $p$ diffuse functions on the heavy atoms and denoted here as maug-ccpVDZ. Basis-set convergence tests are performed with the fully augmented double (aug-cc-pvDZ) and triple (aug-cc-pvTZ) basis sets, in Section S4. In all cases, the exponents of the 
diffuse functions are taken from the corresponding all-electron Dunning's correlation-consistent basis sets. ${ }^{38}$

In the state-specific (energy and variance) optimization runs, we sample a guiding wave function that differs from the current wave function close to the nodes ${ }^{39}$ to guarantee finite variances of the estimators of the gradient, overlap, and Hessian matrix elements. In the state-average energy minimizations, we employ equal weights for the multiple states and sample a guiding wave function constructed as $\Psi_{g}^{2}=\sum_{I}\left|\Psi^{I}\right|^{2}$ to ensure that the distribution sampled has a large overlap with all states of interest. ${ }^{1}$ All wave function parameters (Jastrow, orbital, and CI coefficients) are optimized and the damping factor, $\tau$, in the SR and the Newton method is set to 0.05 and 0.1 , respectively, unless otherwise specified. In the DMC calculations, we treat the pseudopotentials beyond the locality approximation using the T-move algorithm ${ }^{40}$ and employ an imaginary time step of 0.05 a.u. which yields excitation energies converged to better than $0.01 \mathrm{eV}$, as shown in Section S3.

The HF, CIS, and complete-active-space self-consistent-field (CASSCF) calculations are carried out with the program GAMESS(US). ${ }^{41,42}$ For the cyanine dye, we consider different CAS expansions: a $\operatorname{CAS}(6,5)$ and a $\operatorname{CAS}(6,10)$ correlating $6 \pi$ electrons in the orbitals constructed from the $2 \mathrm{p}_{z}$ and $3 \mathrm{p}_{z}$ atomic orbitals and a truncated CAS $(14,13)$ consisting of $6 \pi$ and $8 \sigma$ electrons in 13 bonding and antibonding orbitals. For the retinal model, we employ a minimal $\operatorname{CAS}(6,6)$ active space of $6 \pi$ electrons in the orbitals constructed from the $2 \mathrm{p}_{z}$ atomic orbitals.

The CIPSI calculations are performed with Quantum Package, ${ }^{43}$ and the determinantal expansions are constructed to be eigenstates of $\hat{S}^{2}$. For the cyanine dye where ground and excited states have different symmetries, we follow two paths to construct the CIPSI expansions: (i) We perform separate expansions for the two states starting from the corresponding $\operatorname{CASSCF}(6,10)$ orbitals and match the variances of the CI wave functions to obtain a balanced description of the states. As shown in Table S1, we find that this procedure leads to an automatic match of the second-order perturbation theory (PT2) energy contributions, which are an estimate of the errors of the wave functions with respect to the corresponding full CI (FCI) limit. Using expansions with matched PT2 corrections has recently been shown to lead to accurate QMC excitation energies also for a relatively small number of determinants. $^{9}$ (ii) We perform the expansion of the two states simultaneously, using a common set of orbitals [the excitedstate $\operatorname{CASSCF}(6,10)$ orbitals] and obtain automatically matched PT2 energy corrections during the expansion. ${ }^{9}$ For the retinal model where the ground and excited states have the same symmetry, we have only one set of orbitals for the CIPSI expansions. In this case, we perform a simultaneous expansion with a selection scheme that matches the $\mathrm{CI}$ variances and also attempts to balance the PT2 energy contributions of the two states (see Section S2). ${ }^{44}$

All total energies are computed on the PBE0/cc-pVQZ ground-state geometry of the cyanine ${ }^{45}$ and retinal molecules. The DFT geometry optimization of the retinal model is performed with the program Gaussian. ${ }^{46}$ The coupled cluster results are obtained with Psi4. ${ }^{47}$

\section{RESULTS}

We compute the lowest $\pi \rightarrow \pi^{*}$ vertical excitation energy of the cyanine dye $\left(\mathrm{C}_{3} \mathrm{H}_{3}\left(\mathrm{NH}_{2}\right)_{2}^{+}\right)$and the minimal model of the retinal protonated Schiff base $\left(\mathrm{C}_{5} \mathrm{H}_{6} \mathrm{NH}_{2}^{+}\right)$, depicted in Figure 1 and denoted as CN5 and PSB3, respectively. As already mentioned, besides being generally challenging for electronic structure methods, ${ }^{4,25-28}$ these examples are representative of the two cases of a ground (S0) state and an excited (S1) state of different (CN5) and equal (PSB3) symmetries. Correspondingly, the energy minimization scheme is state-specific for CN5 and state-average for PSB3, while variance minimization affords a state-specific optimization for both molecules, at least in principle.

4.1. Ground and Excited States of Different Symmetry. In Table 1, we list the ground- and excited-state energies and corresponding excitation energies of CN5 computed in VMC and DMC with different wave functions optimized by (state-specific) energy minimization. The simplest case consists of a single determinant (HF) and a HOMO-LUMO (HL) two-determinant wave function for the ground and excited states, respectively. We then consider CIS expansions, CAS expansions with increasing active spaces, and balanced CIPSI expansions with different choices of the starting orbitals, namely, independent sets for the two states (CIPSI-SS) or a common set of orbitals $\left(\right.$ CIPSI-B $\left.{ }_{1}\right)$. The excitation energies are displayed in Figure 2.

The general trend is a decrease in excitation energy toward the extrapolated full $\mathrm{CI}$ (exFCI) and approximate coupled cluster singles, doubles, and triples model (CC3) reference values for better wave functions. As an exception, when we move from the HF/HL to CIS wave functions, the VMC energies of both states decrease but the corresponding excitation energy becomes worse. With increasingly large CAS expansions, both the total and the excitation energies improve but the convergence is very slow. For all these wave functions, the DMC excitation energy is lower than the VMC value and becomes within $0.1 \mathrm{eV}$ of the reference results for the largest active spaces with about 50,000 and 70,000 determinants for the ground and excited states, respectively. By comparison, the errors of TDDFT and CASPT2 can be as large as 0.4 and $-0.2 \mathrm{eV}$, respectively. ${ }^{4,45}$

The quality of the results exhibits a further, dramatic improvement with the use of CIPSI expansions. The VMC and DMC energies obtained with the smallest CIPSI wave function are lower than the corresponding values obtained with the largest CAS considered here. Furthermore, constructing ground- and excited-state CIPSI expansions with similar PT2 corrections leads to a balanced description of both states and to VMC excitation energies which change very little with increasing expansion size, being irregularly scattered over a small energy range of $0.08 \mathrm{eV}$. Importantly, the DMC excitation energies are compatible with the VMC ones and in excellent agreement with the CC3 and exFCI values. Finally, employing two different sets of orbitals to generate the CIPSI expansions leads to marginal differences, namely, to DMC

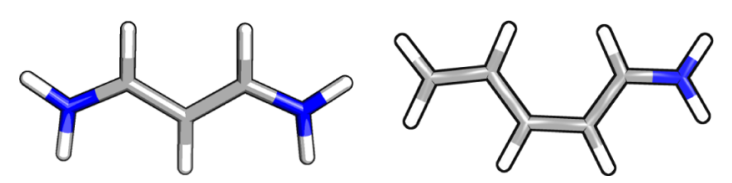

Figure 1. Schematic representations of the CN5 (left) and PSB3 (right) molecules. White, gray, and blue denote hydrogen, carbon, and nitrogen, respectively. 
Table 1. VMC and DMC Total Energies (a.u.) and Excitation Energies ( $\Delta E, \mathrm{eV})$ of CN5 Obtained for Different Wave Functions Optimizing All Parameters (Jastrow, Orbital, and CI Coefficients) in Energy Minimization

\begin{tabular}{|c|c|c|c|c|c|c|c|c|c|c|}
\hline \multirow[b]{2}{*}{ WF } & \multicolumn{2}{|c|}{ no. det } & \multicolumn{2}{|c|}{ no. param } & \multicolumn{3}{|c|}{ VMC } & \multicolumn{3}{|c|}{$\mathrm{DMC}$} \\
\hline & So & S1 & So & S1 & $E(\mathrm{~S} 0)$ & $E(\mathrm{~S} 1)$ & $\Delta E$ & $E(\mathrm{~S} 0)$ & $E(\mathrm{~S} 1)$ & $\Delta E$ \\
\hline $\mathrm{HF} / \mathrm{HL}$ & 1 & 2 & 516 & 529 & $-40.8372(4)$ & $-40.6460(3)$ & $5.202(14)$ & $-40.9378(3)$ & $-40.7509(3)$ & $5.086(11)$ \\
\hline $\mathrm{HF} / \mathrm{CIS}$ & 1 & 980 & 516 & 4751 & $-40.8372(4)$ & $-40.6505(3)$ & $5.080(14)$ & $-40.9378(3)$ & $-40.7533(3)$ & $5.020(11)$ \\
\hline CIS & 999 & 980 & 5260 & 4751 & $-40.8444(4)$ & $-40.6505(3)$ & $5.278(14)$ & $-40.9393(3)$ & $-40.7533(3)$ & $5.061(11)$ \\
\hline $\operatorname{CAS}(6,5)$ & 52 & 48 & 567 & 561 & $-40.8468(4)$ & $-40.6583(4)$ & $5.130(15)$ & $-40.9433(3)$ & $-40.7582(2)$ & $5.038(10)$ \\
\hline $\operatorname{CAS}(6,10)$ & 7232 & 7168 & 3134 & 3064 & $-40.8498(4)$ & $-40.6628(4)$ & $5.090(15)$ & $-40.9439(3)$ & $-40.7594(3)$ & $5.022(11)$ \\
\hline $\operatorname{CAS}(14,13)$ & 48,206 & 72,732 & 9480 & 11,727 & $-40.8583(3)$ & $-40.6713(3)$ & $5.091(10)$ & $-40.9442(7)$ & $-40.7611(7)$ & $4.983(26)$ \\
\hline \multirow[t]{4}{*}{ CIPSI-SS } & 376 & 1094 & 1567 & 2609 & $-40.8646(3)$ & $-40.6842(3)$ & $4.908(12)$ & $-40.9467(3)$ & $-40.7665(3)$ & $4.905(10)$ \\
\hline & 1344 & 4382 & 2478 & 4531 & $-40.8798(3)$ & $-40.7013(3)$ & $4.857(13)$ & $-40.9502(2)$ & $-40.7711(2)$ & $4.872(09)$ \\
\hline & 2460 & 8782 & 3555 & 6561 & $-40.8896(3)$ & $-40.7099(3)$ & $4.890(12)$ & $-40.9532(2)$ & $-40.7748(2)$ & $4.856(09)$ \\
\hline & 3913 & 14,114 & 4842 & 8312 & $-40.8941(2)$ & $-40.7167(3)$ & $4.828(11)$ & $-40.9559(2)$ & $-40.7775(2)$ & $4.856(08)$ \\
\hline \multirow[t]{2}{*}{ CIPSI-B ${ }_{1}$} & 2456 & 6120 & 3971 & 5466 & $-40.8847(2)$ & $-40.7053(2)$ & $4.880(09)$ & $-40.9521(2)$ & $-40.7727(2)$ & $4.881(09)$ \\
\hline & 4829 & 13,130 & 5737 & 8021 & $-40.8945(3)$ & $-40.7150(3)$ & $4.889(13)$ & $-40.9560(2)$ & $-40.7766(2)$ & $4.882(08)$ \\
\hline exFCI/aug-cc-pVDZ ${ }^{48}$ & & & & & & & & & & 4.89 \\
\hline CC3/aug-cc-pVDZ & & & & & & & & & & 4.851 \\
\hline CC3/aug-cc-pVTZ & & & & & & & & & & 4.844 \\
\hline
\end{tabular}

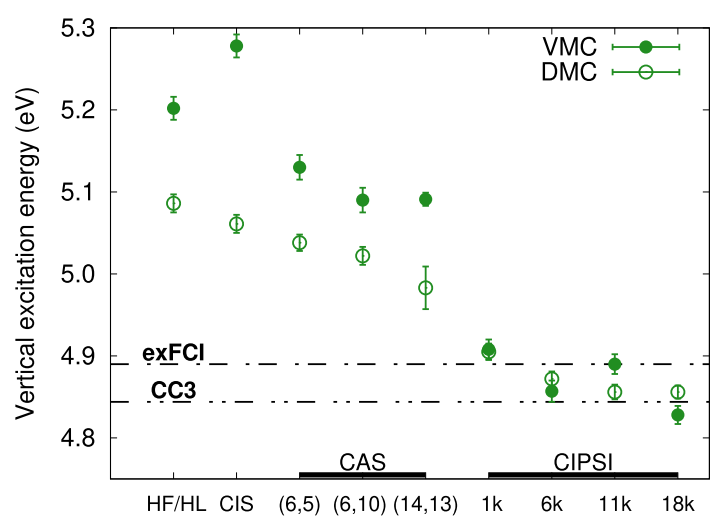

Figure 2. VMC and DMC excitation energies of CN5 calculated with different wave functions optimized in energy minimization. The exFCI/aug-cc-pVDZ ${ }^{48}$ and CC3/aug-cc-pVTZ reference values are also shown. The approximate total number of determinants for the CIPSI-SS wave functions of the ground and excited states is indicated. excitation energies of $4.856(8)$ and $4.882(8) \mathrm{eV}$, which are both bracketed by the reference values.

Having verified that state-specific energy optimization in combination with accurate wave functions allows the robust treatment of CN5, we now employ variance minimization with the $\sigma_{\omega}^{2}$ functional to optimize the $\operatorname{CAS}(6,5)$ and $\operatorname{CAS}(6,10)$ wave functions of the ground and excited states. The convergence of the corresponding VMC variances and energies is shown in Figure 3. For the smaller CAS(6,5), we observe that while the variance converges rather quickly, the energy appears to do so more slowly and only after undershooting to a value which generally depends on the statistical error and initial conditions of the run. For an approximate wave function, the optimal parameters in variance minimization may differ from those obtained in energy minimization. Therefore, during the optimization of the variance, the energy can become lower than the final one.

As reported in Table 2, the optimal ground- and excitedstate energies are higher by about $30 \mathrm{mHartree}$ than the
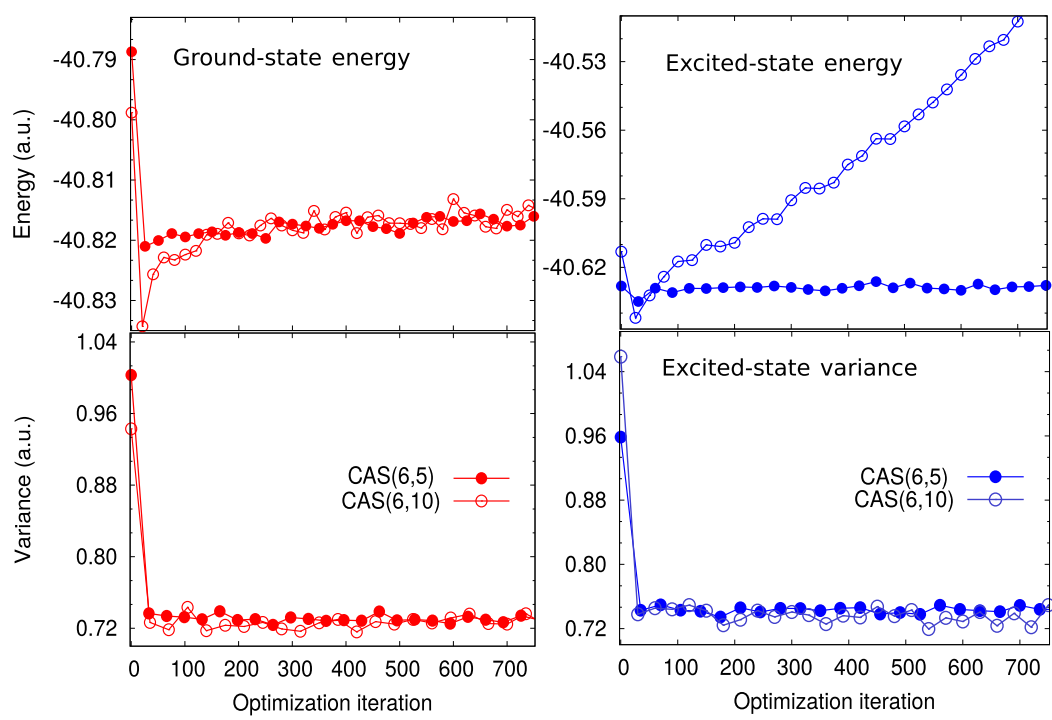

Figure 3. Convergence of the VMC energy (top) and variance (bottom) of the ground (left) and excited (right) states of CN5 in the optimization of the $\operatorname{CAS}(6,5)$ and $\operatorname{CAS}(6,10)$ wave functions in variance minimization. 
Table 2. VMC Energies and Variances (a.u.) and Vertical Excitation Energies (eV) of CN5 Obtained with Energy and Variance Minimization

\begin{tabular}{|c|c|c|c|c|c|c|c|c|c|c|}
\hline & \multicolumn{5}{|c|}{ energy min. } & \multicolumn{5}{|c|}{ variance min. } \\
\hline & $E(\mathrm{~S} 0)$ & $E(\mathrm{~S} 1)$ & $\Delta E$ & $\sigma^{2}(\mathrm{~S} 0)$ & $\sigma^{2}(\mathrm{~S} 1)$ & $E(\mathrm{~S} 0)$ & $E(\mathrm{~S} 1)$ & $\Delta E$ & $\sigma^{2}(\mathrm{~S} 0)$ & $\sigma^{2}(\mathrm{~S} 1)$ \\
\hline $\operatorname{CAS}(6,5)$ & $-40.8468(4)$ & $-40.6583(4)$ & $5.13(1)$ & 0.862 & 0.885 & $-40.8170(5)$ & $-40.6270(5)$ & $5.17(2)$ & 0.733 & 0.743 \\
\hline $\operatorname{CAS}(6,10)$ & $-40.8498(4)$ & $-40.6628(4)$ & $5.09(1)$ & 0.855 & 0.868 & $-40.8163(4)$ & & & 0.731 & \\
\hline
\end{tabular}
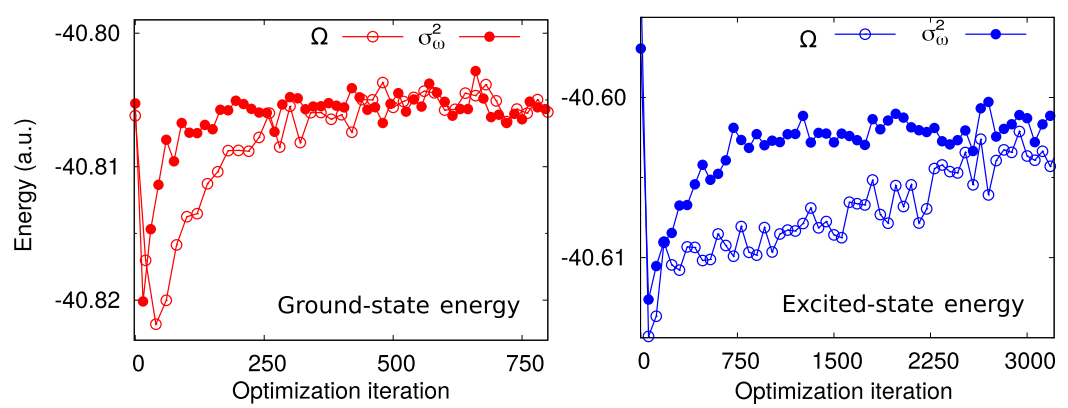

Figure 4. Convergence of VMC energy of the ground (left) and excited (right) states of CN5 in the optimization of the HF/HL wave functions within variance minimization with the $\sigma_{\omega}^{2}$ (our default) and the $\Omega$ functional.

Table 3. VMC and DMC Total Energies (a.u.) and Excitation Energies ( $\Delta E$, eV) of PSB3 Obtained for Different Wave Functions Optimizing All Parameters (Jastrow, Orbital, and CI Coefficients) in Energy Minimization

\begin{tabular}{|c|c|c|c|c|c|c|c|c|}
\hline \multirow[b]{2}{*}{ WF } & \multirow[b]{2}{*}{ no. det } & \multirow[b]{2}{*}{ no. param } & \multicolumn{3}{|c|}{ VMC } & \multicolumn{3}{|c|}{$\mathrm{DMC}$} \\
\hline & & & $E(\mathrm{~S} 0)$ & $E(\mathrm{~S} 1)$ & $\Delta E$ & $E(\mathrm{~S} 0)$ & $E(\mathrm{~S} 1)$ & $\Delta E$ \\
\hline $\operatorname{CAS}(6,6)$ & 400 & 1645 & $-42.8091(2)$ & $-42.6471(2)$ & $4.409(9)$ & $-42.9118(2)$ & $-42.7541(2)$ & $4.293(6)$ \\
\hline CIPSI & 422 & 4011 & $-42.8174(2)$ & $-42.6623(2)$ & $4.221(9)$ & $-42.9133(2)$ & $-42.7578(2)$ & $4.233(6)$ \\
\hline & 1158 & 5968 & $-42.8297(2)$ & $-42.6735(2)$ & $4.252(9)$ & $-42.9160(2)$ & $-42.7609(2)$ & $4.221(6)$ \\
\hline & 2579 & 8106 & $-42.8357(2)$ & $-42.6796(2)$ & $4.247(9)$ & $-42.9169(2)$ & $-42.7621(2)$ & $4.214(6)$ \\
\hline CC3/aug-cc-pVDZ & & & & & & & & 4.19 \\
\hline CC3/aug-cc-pVTZ & & & & & & & & 4.16 \\
\hline
\end{tabular}

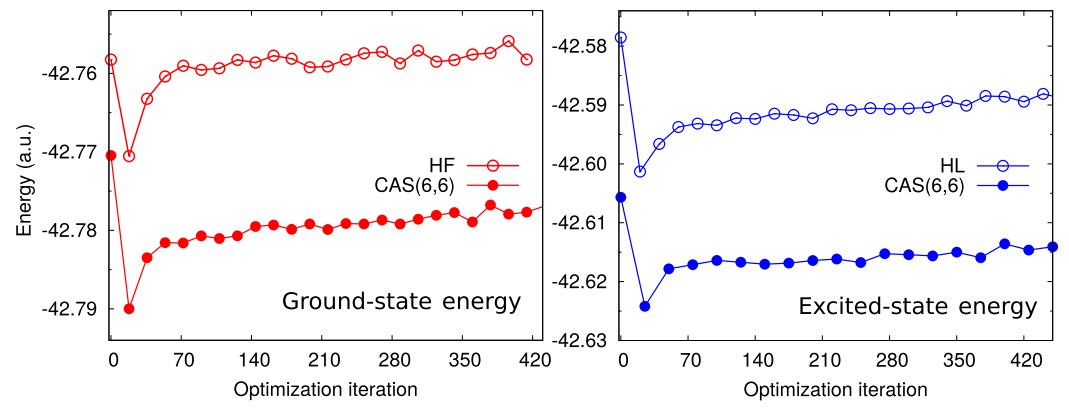

Figure 5. Convergence of the VMC energy of the ground (red) and excited (blue) states of PSB3 in the optimization of the RHF/HL and CAS $(6,6)$ wave functions within variance minimization.

corresponding values obtained in energy minimization but the resulting excitation energy is compatible within the statistical error.

If we move to the larger $\operatorname{CAS}(6,10)$ determinantal expansion, we find, however, that while the variance reaches a stable value and the ground-state energy has a similar behavior to the $\operatorname{CAS}(6,5)$ case, the energy of the excited state grows steadily and it is therefore not possible to estimate the vertical excitation energy of the system. Surprisingly, even in the simplest case of the one-configuration ( $\mathrm{HF} / \mathrm{HL}$ ) wave functions, the energy of the excited state keeps increasing slowly even after 600 iterations, as shown in Figure 4, while the ground-state energy behaves similarly to the corresponding CAS cases.
Importantly, the apparently unstable behavior is independent of the initial value of $\omega$ and the number of steps over which we keep $\omega$ fixed (see Section S5). The use of smaller or larger damping factors (i.e., $\tau=0.04$ and 0.2 ) leads to the same pathological growth of the excited-state energy, characterized by the same slope as a function of time, as shown in Figure S4. Moreover, we recover the same behavior also when using a gradient-only-based optimizer (see Figure S5). Finally, minimizing the $\Omega$ functional instead of $\sigma_{\omega}^{2}$ yields an excitedstate energy which ultimately increases with iterations, as shown for the excited-state HL wave function in Figure 4.

4.2. Ground and Excited States of the Same Symmetry. For PSB3, we optimize the wave functions in energy minimization in a state-average fashion and report the resulting VMC and DMC total energies and vertical excitation 
energies in Table 3. As in the CN5 case, CIPSI wave functions are superior to CAS expansions of similar size, and with only about 400 determinants, the use of CIPSI yields not only lower total energies but also a VMC vertical excitation energy, in good agreement with the CC3 reference, largely correcting the error of $0.25 \mathrm{eV}$ obtained with the $\operatorname{CAS}(6,6)$ wave function. For all CIPSI expansions, the DMC excitation energies are always quite close to the correspondent VMC results and, for the larger expansions, within $0.05 \mathrm{eV}$ of the CC3 value.

When we perform state-specific variance minimization, we encounter great difficulties in the convergence of the energies, as we show for the $\mathrm{HF} / \mathrm{HL}$ and $\operatorname{CAS}(6,6)$ wave functions in Figure 5. Different from CN5, we find, in general, that not only the energy of the excited state but also that of the ground state grows steadily with the iteration number.

\section{DISCUSSION AND CONCLUSIONS}

While our results confirm the high accuracy reachable in QMC with energy minimization, they evidence severe problems in variance minimization which, in most cases, preclude the estimation of the excitation energy. To gain a better understanding of the troublesome behavior of the energy during variance minimization, we further investigate the simple case of the HL wave function of CN5 (Figure 4) and find that the energy of the state drifts to higher values during variance minimization also when one optimizes only the LUMO orbital. Therefore, because optimization of an orbital can be achieved by mixing it with the unoccupied ones of the same symmetry, we can recast the LUMO optimization into the linear variation of the CI coefficients of the single excitations out of the LUMO orbital, which amount to only 12 additional CSFs in our basis set. With such a small expansion, we can then diagonalize the Hamiltonian on the basis of the CSFs times the Jastrow factor to estimate its 13 eigenvalues and eigenvectors and work directly on the basis of the eigenstates to assess the behavior of variance minimization when starting from the states which are optimal for energy minimization.

In Figure 6, we show the evolution of the VMC variance and energy for four variance minimization runs in which we start from different eigenvectors, taking the corresponding eigenvalues as the initial target energies $\omega$. In particular, we consider the lowest state in $\mathrm{B}_{1}$ symmetry and the second, fourth, and thirteenth (corresponding to the highest energy) states. We note that because our states are not exact eigenstates of the full Hamiltonian, the corresponding variances of the local energy are non-zero and are spread over about 0.5 a.u. with the lowest value in correspondence to the second state. In principle, one would expect to find a feature of the variance landscapeideally a local minimum-near each of the approximate eigenstates because the functionals $\sigma_{\omega}^{2}$ and $\Omega$ are designed to select a particular state through the initial value of $\omega$ and minimize the variance of this state. Here, the selection of the state is further facilitated starting each run precisely from the chosen eigenstate, and variance minimization should perform minor adjustments of the initial parameters from their optimal values for energy.

The behavior illustrated in Figure 6 is totally different, with all optimization runs leaking down to successive lower-variance states and eventually converging to the absolute minimum corresponding to the second eigenstate. The staircase shape of the variance evolution points to the presence of flat regions of the variance landscape close to the eigenstates, from which the optimization can eventually escape. This is further corrobo-

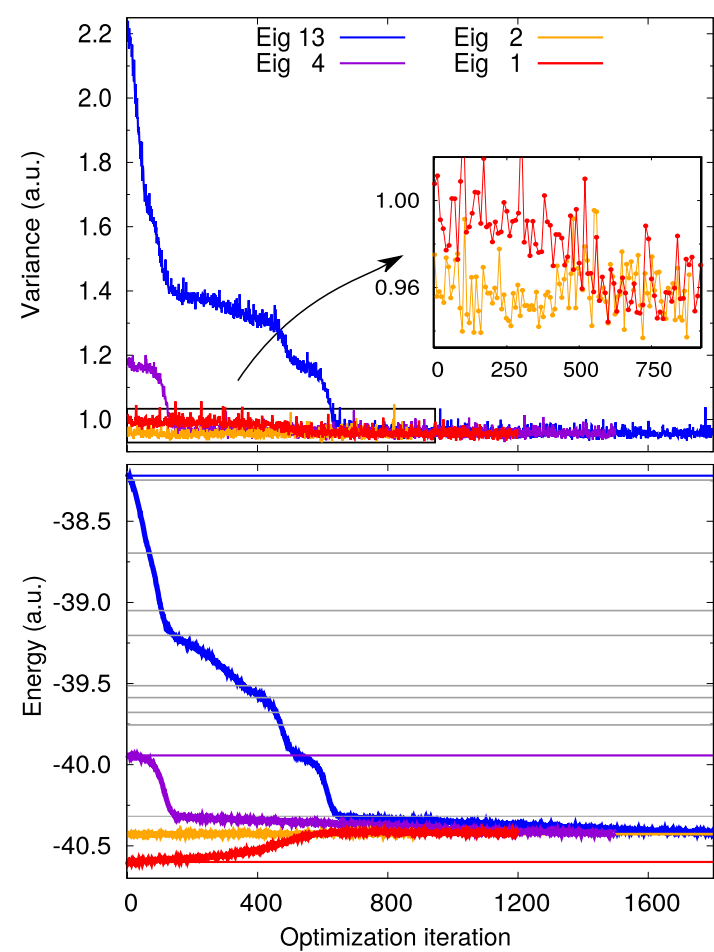

Figure 6. Convergence of the VMC variance (top) and energy (bottom) of CN5 in the CI optimization of a small expansion (see text) with variance minimization. The horizontal lines in the energy plot correspond to the eigenvalues in this reduced space, and the colored ones are the eigenstates used as the starting point in four optimization runs. The damping factor used in the Newton method is $\tau=0.2$.

rated if we follow the evolution of the CI coefficients, as shown starting from the highest-energy state in Figure 7: the initial coefficient quickly decreases to zero and other eigenstates become populated until convergence on the second state. In proximity of some eigenstates, the variance displays a more pronounced plateau, where the system spends enough time to acquire the full character of this particular state. It is also interesting to note that the states are populated sequentially with the order determined by decreasing energies. We stress

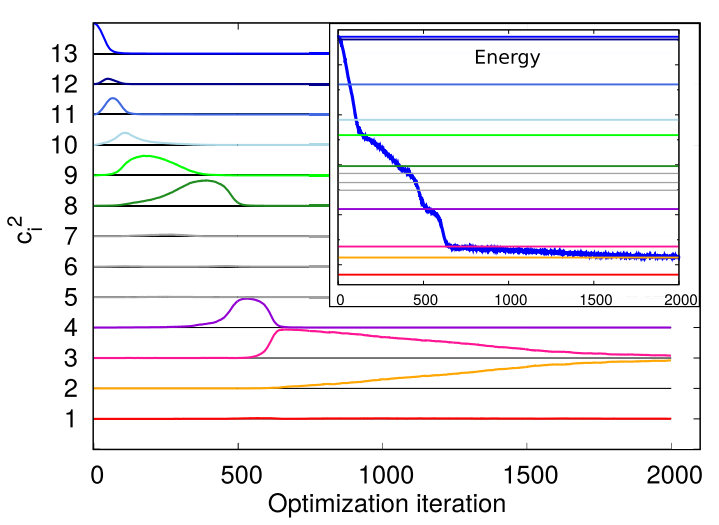

Figure 7. Evolution of the square of the CI coefficients $c_{i}^{2}$ (offset by $i$ for clarity) of the small expansion of $\mathrm{CN} 5$ during variance minimization, for the run starting from the 13th eigenvector; in the inset, the evolution of the energy is replicated to emphasize flat regions in the energy landscape close to an eigenstate (i.e., when the corresponding $c_{i} \approx 1$ ). 

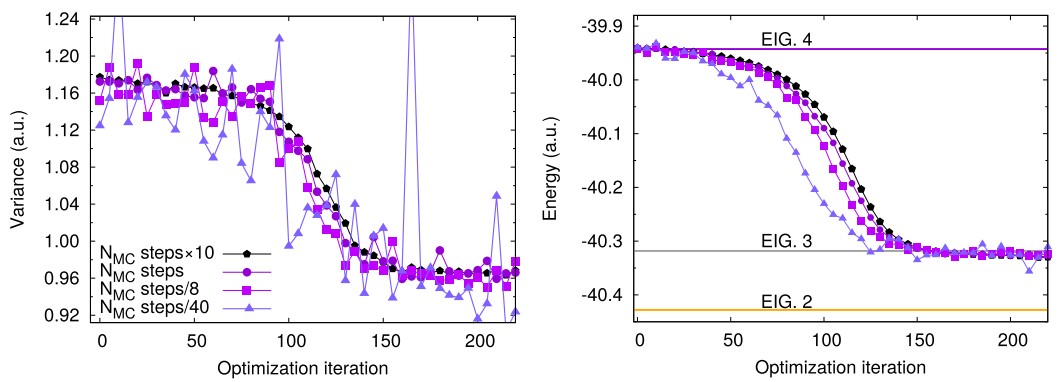

Figure 8. Convergence of the variance (left) and energy (right) for different lengths of the Monte Carlo runs used to compute the gradient and Hessian matrix during optimization, starting from the 4 th eigenvector. $N_{\mathrm{MC}}$ is the number of Monte Carlo steps used in Figure 6.
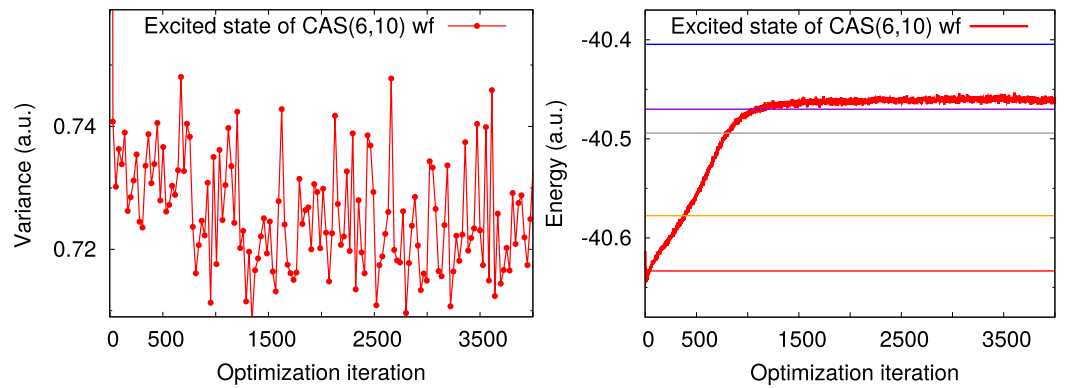

Figure 9. Variance (left) and energy (right) convergence for the optimization of the excited state of the CAS $(6,10)$ wave function. The horizontal lines in the energy plot correspond to the first eigenvalue roots obtained with the Davidson optimization.

that we observe a similar behavior of the variance also when using the $\Omega$ functional starting from the same set of approximate eigenstates (see Figure S6).

In Figure 8, we investigate the impact of the statistical error on the loss of the selected state. In particular, we focus on the evolution of the variance and the energy starting from the 4th eigenvector for different lengths of the VMC runs used to compute the gradient and Hessian matrix. The shortest run (larger statistical error) loses the target state in a slightly smaller number of steps. However, the other runs give very similar results, suggesting that even longer VMC runs would not stabilize the target state.

This simple wave function of CN5 is an explicit instance of missing one-to-one correspondence between minima of the variance and approximate eigenstates. Even if the actual number of minima and their correspondence to particular eigenstates remain unknown, in general, the understanding gained here clearly applies to the behavior that we have observed for more complicated wave functions. As an explicit example, we revisit the very problematic optimization of the excited-state CAS $(6,10)$ wave function (Figure 3 ) and perform a much longer calculation, finding that the energy eventually converges, as shown in Figure 9. For the final set of Jastrow and orbital parameters, we determine the eigenvalues in the linear space of the determinants times the Jastrow factor and recover a similar behavior to what was observed in the simple example: the minimization of $\sigma_{\omega}{ }^{2}$ brings the system approximately to an eigenstate with a lower variance, which is, in this case, the 4 th one.

By systematically improving the wave function, it is possible, in principle, to approach the exact eigenstate and its zerovariance property, thus recovering the corresponding minimum in the variance landscape. However, a CIPSI expansion which gives excellent results in energy minimization does not always prove sufficient to stabilize variance minimization (see Section S9). In general, going to extended determinantal expansions for the sake of a stable variance minimization, when energy minimization results are already satisfactory, appears unpractical, if feasible at all.

In summary, we have shown that the combination of energy minimization with an appropriate choice of the ground- and excited-state wave functions via a balanced CIPSI procedure leads to excitation energies that are in excellent agreement already at the VMC level with the reference values. In particular, we obtained a robust convergence of the total ground- and excited-state energies and a very accurate excitation energy not only in the easier state-specific case of CN5 but also when employing energy minimization in a stateaverage fashion for PSB3. On the other hand, we encountered severe problems when employing variance minimization because over sufficiently long optimization runs, one may lose the state of interest in favor of a state with lower variance, as we clearly demonstrated with a simple but realistic example. Even though, theoretically, the functionals $\sigma_{\omega}{ }^{2}$ and $\Omega$ have a built-in possibility to target the energy of a specific state, in practice, this is generally not sufficient to maintain the parameters close to the desired local minimum of the variance. Therefore, these considerations lead to the conclusion that with the present functionals and no a priori knowledge of the parameter landscape of the variance for the system of interest, energy minimization is a safer and more stable procedure.

\section{ASSOCIATED CONTENT}

\section{Supporting Information}

The Supporting Information is available free of charge at https://pubs.acs.org/doi/10.1021/acs.jctc.0c00147.

Derivation and discussion of the expressions of the gradient and approximate Hessian of the variance; CIPSI energies for various expansions; basis-set dependence of the VMC and DMC excitation energies; DMC excitation energy versus time step; dependence of variance minimization on the choice of $\omega$, number of 
steps with $\omega$ fixed, and damping factor in the Newton method; optimizations with a gradient-based optimizer and with the $\Omega$ functional; and variance minimizations with CIPSI wave functions (PDF)

\section{AUTHOR INFORMATION}

\section{Corresponding Authors}

Anthony Scemama - Laboratoire de Chimie et Physique Quantiques, Universite de Toulouse, CNRS, UPS, 31062 Toulouse Cedex 09, France; Email: scemama@irsamc.upstlse.fr

Wim J. Briels - MESA+ Institute for Nanotechnology, University of Twente, 7500 AE Enschede, The Netherlands; Email: w.j.briels@utwente.nl

Saverio Moroni - CNR-IOM DEMOCRITOS, Istituto Officina dei Materiali, I-34136 Trieste, Italy; SISSA Scuola Internazionale Superiore di Studi Avanzati, I-34136 Trieste, Italy; Email: moroni@democritos.it

Claudia Filippi - MESA+ Institute for Nanotechnology, University of Twente, 7500 AE Enschede, The Netherlands; 이이.org/0000-0002-2425-6735; Email: c.filippi@ utwente.nl

\section{Author}

Alice Cuzzocrea - MESA+ Institute for Nanotechnology, University of Twente, 7500 AE Enschede, The Netherlands; (1) orcid.org/0000-0001-7446-9643

Complete contact information is available at: https://pubs.acs.org/10.1021/acs.jctc.0c00147

\section{Notes}

The authors declare no competing financial interest.

\section{ACKNOWLEDGMENTS}

A.C. is supported by the "Computational Science for Energy Research and Netherlands eScience Center joint program" (project CSER.JCER.022) of the Netherlands Organisation for Scientific Research (NWO). This work was carried out on the Dutch national supercomputer Cartesius with the support of SURF Cooperative.

\section{REFERENCES}

(1) Filippi, C.; Zaccheddu, M.; Buda, F. Absorption spectrum of the green fluorescent protein chromophore: a difficult case for ab initio methods? J. Chem. Theory Comput. 2009, 5, 2074-2087.

(2) Zimmerman, P. M.; Toulouse, J.; Zhang, Z.; Musgrave, C. B.; Umrigar, C. J. Excited states of methylene from quantum Monte Carlo. J. Chem. Phys. 2009, 131, 124103.

(3) Valsson, O.; Filippi, C. Photoisomerization of model retinal chromophores: insight from quantum Monte Carlo and multiconfigurational perturbation theory. J. Chem. Theory Comput. 2010, $6,1275-1292$.

(4) Send, R.; Valsson, O.; Filippi, C. Electronic Excitations of Simple Cyanine Dyes: Reconciling Density Functional and Wave Function Methods. J. Chem. Theory Comput. 2011, 7, 444-455.

(5) Valsson, O.; Campomanes, P.; Tavernelli, I.; Rothlisberger, U.; Filippi, C. Rhodopsin absorption from first principles: Bypassing common pitfalls. J. Chem. Theory Comput. 2013, 9, 2441-2454.

(6) Guareschi, R.; Zulfikri, H.; Daday, C.; Floris, F. M.; Amovilli, C.; Mennucci, B.; Filippi, C. Introducing QMC/MMpol: Quantum Monte Carlo in Polarizable Force Fields for Excited States. J. Chem. Theory Comput. 2016, 12, 1674-1683.
(7) Hunt, R. J.; Szyniszewski, M.; Prayogo, G. I.; Maezono, R.; Drummond, N. D. Quantum Monte Carlo calculations of energy gaps from first principles. Phys. Rev. B 2018, 98, 075122.

(8) Blunt, N. S.; Neuscamman, E. Excited-State Diffusion Monte Carlo Calculations: A Simple and Efficient Two-Determinant Ansatz. J. Chem. Theory Comput. 2019, 15, 178-189.

(9) Dash, M.; Feldt, J.; Moroni, S.; Scemama, A.; Filippi, C. Excited States with Selected Configuration Interaction-Quantum Monte Carlo: Chemically Accurate Excitation Energies and Geometries. J. Chem. Theory Comput. 2019, 15, 4896-4906.

(10) Foulkes, W. M. C.; Mitas, L.; Needs, R. J.; Rajagopal, G. Quantum Monte Carlo simulations of solids. Rev. Mod. Phys. 2001, $73,33-83$.

(11) Lüchow, A. Quantum Monte Carlo methods. Wiley Interdiscip. Rev.: Comput. Mol. Sci. 2011, 1, 388-402.

(12) Austin, B. M.; Zubarev, D. Y.; Lester, W. A. Quantum Monte Carlo and Related Approaches. Chem. Rev. 2012, 112, 263-288.

(13) Sorella, S.; Capriotti, L. Algorithmic differentiation and the calculation of forces by quantum Monte Carlo. J. Chem. Phys. 2010, 133, 234111.

(14) Neuscamman, E.; Umrigar, C. J.; Chan, G. K.-L. Optimizing large parameter sets in variational quantum Monte Carlo. Phys. Rev. B: Condens. Matter Mater. Phys. 2012, 85, 045103.

(15) Filippi, C.; Assaraf, R.; Moroni, S. Simple formalism for efficient derivatives and multi-determinant expansions in quantum Monte Carlo. J. Chem. Phys. 2016, 144, 194105.

(16) Assaraf, R.; Moroni, S.; Filippi, C. Optimizing the Energy with Quantum Monte Carlo: A Lower Numerical Scaling for JastrowSlater Expansions. J. Chem. Theory Comput. 2017, 13, 5273-5281.

(17) Dash, M.; Moroni, S.; Scemama, A.; Filippi, C. Perturbatively Selected Configuration-Interaction Wave Functions for Efficient Geometry Optimization in Quantum Monte Carlo. J. Chem. Theory Comput. 2018, 14, 4176-4182.

(18) Coldwell, R. L. Zero Monte Carlo error or quantum mechanics is easier. Int. J. Quantum Chem. 2009, 12, 215-222.

(19) Umrigar, C. J.; Wilson, K. G.; Wilkins, J. W. Optimized Trial Wave Function for Quantum Monte Carlo Calculations. Phys. Rev. Lett. 1988, 60, 1719-1722.

(20) Malatesta, A.; Fahy, S.; Bachelet, G. B. Variational quantum Monte Carlo calculation of the cohesive properties of cubic boron nitride. Phys. Rev. B: Condens. Matter Mater. Phys. 1997, 56, 1220112210 .

(21) Kent, P. R. C.; Needs, R. J.; Rajagopal, G. Monte Carlo energy and variance-minimization techniques for optimizing many-body wave functions. Phys. Rev. B: Condens. Matter Mater. Phys. 1999, 59, 12344-12351.

(22) Umrigar, C. J.; Filippi, C. Energy and Variance Optimization of Many-Body Wave Functions. Phys. Rev. Lett. 2005, 94, 150201.

(23) Shea, J. A. R.; Neuscamman, E. Size Consistent Excited States via Algorithmic Transformations between Variational Principles. J. Chem. Theory Comput. 2017, 13, 6078-6088.

(24) Pineda Flores, S. D.; Neuscamman, E. Excited State Specific Multi-Slater Jastrow Wave Functions. J. Phys. Chem. A 2019, 123, 1487-1497.

(25) Valsson, O.; Angeli, C.; Filippi, C. Excitation energies of retinal chromophores: critical role of the structural model. Phys. Chem. Chem. Phys. 2012, 14, 11015-11020.

(26) Huix-Rotllant, M.; Filatov, M.; Gozem, S.; Schapiro, I.; Olivucci, M.; Ferré, N. Assessment of Density Functional Theory for Describing the Correlation Effects on the Ground and Excited State Potential Energy Surfaces of a Retinal Chromophore Model. J. Chem. Theory Comput. 2013, 9, 3917-3932.

(27) Tuna, D.; Lefrancois, D.; Wolański, Ł.; Gozem, S.; Schapiro, I.; Andruniów, T.; Dreuw, A.; Olivucci, M. Assessment of Approximate Coupled-Cluster and Algebraic-Diagrammatic-Construction Methods for Ground- and Excited-State Reaction Paths and the ConicalIntersection Seam of a Retinal-Chromophore Model. J. Chem. Theory Comput. 2015, 11, 5758-5781. 
(28) Le Guennic, B.; Jacquemin, D. Taking Up the Cyanine Challenge with Quantum Tools. Acc. Chem. Res. 2015, 48, 530-537.

(29) As the Jastrow factor, we use the exponential of the sum of two fifth-order polynomials of the electron-nuclear and the electronelectron distances, and rescale the inter-particle distances as $R=(1-$ $\exp (-\kappa r)) / \kappa$ with $\kappa$ set to 0.6 a.u. We employ different electronnucleus Jastrow factors to describe the correlation of an electron with $\mathrm{H}, \mathrm{C}$, and $\mathrm{N}$. The total number of free parameters to be optimized in the Jastrow factor is 17 for the systems considered here.

(30) Neuscamman, E. Communication: Variation after response in quantum Monte Carlo. J. Chem. Phys. 2016, 145, 081103.

(31) Sorella, S.; Casula, M.; Rocca, D. Weak binding between two aromatic rings: Feeling the van der Waals attraction by quantum Monte Carlo methods. J. Chem. Phys. 2007, 127, 014105.

(32) Sabzevari, I.; Mahajan, A.; Sharma, S. An accelerated linear method for optimizing non-linear wavefunctions in variational Monte Carlo. J. Chem. Phys. 2020, 152, 024111.

(33) Press, W. H.; Teukolsky, S. A.; Vetterling, W. T.; Flannery, B. P. Numerical Recipes, 3rd ed; The Art of Scientific Computing; Cambridge University Press: The Edinburgh Building, Cambridge, United Kingdom, 2007; pp 801-805.

(34) Toulouse, J.; Umrigar, C. J. Full optimization of Jastrow-Slater wave functions with application to the first-row atoms and homonuclear diatomic molecules. J. Chem. Phys. 2008, 128, 174101.

(35) CHAMP is a quantum Monte Carlo program package written by C. J. Umrigar, C. Filippi, S. Moroni and collaborators.

(36) Burkatzki, M.; Filippi, C.; Dolg, M. Energy-consistent pseudopotentials for quantum Monte Carlo calculations. J. Chem. Phys. 2007, 126, 234105.

(37) Dolg, M.; Filippi, C. For the Hydrogen Atom, We Use a More Accurate BFD Pseudopotential and Basis Set, private communication. 2015

(38) Kendall, R. A.; Dunning, T. H., Jr; Harrison, R. J. Electron affinities of the first-row atoms revisited. Systematic basis sets and wave functions. J. Chem. Phys. 1992, 96, 6796-6806.

(39) Attaccalite, C.; Sorella, S. Stable liquid hydrogen at high pressure by a novel ab initio molecular-dynamics calculation. Phys. Rev. Lett. 2008, 100, 114501.

(40) Casula, M. Beyond the locality approximation in the standard diffusion Monte Carlo method. Phys. Rev. B: Condens. Matter Mater. Phys. 2006, 74, 161102.

(41) Schmidt, M. W.; Baldridge, K. K.; Boatz, J. A.; Elbert, S. T.; Gordon, M. S.; Jensen, J. H.; Koseki, S.; Matsunaga, N.; Nguyen, K. A.; Su, S.; Windus, T. L.; Dupuis, M.; Montgomery, J. A., Jr General atomic and molecular electronic structure system. J. Comput. Chem. 1993, 14, 1347-1363.

(42) Gordon, M. S.; Schmidt, M. W. In Theory and Applications of Computational Chemistry; Dykstra, C. E., Frenking, G., Kim, K. S., Scuseria, G. E., Eds.; Elsevier: Amsterdam, 2005; pp 1167-1189.

(43) Garniron, Y.; Applencourt, T.; Gasperich, K.; Benali, A.; Ferté, A.; Paquier, J.; Pradines, B.; Assaraf, R.; Reinhardt, P.; Toulouse, J.; Barbaresco, P.; Renon, N.; David, G.; Malrieu, J.-P.; Véril, M.; Caffarel, M.; Loos, P.-F.; Giner, E.; Scemama, A. Quantum Package 2.0: An Open-Source Determinant-Driven Suite of Programs. J. Chem. Theory Comput. 2019, 15, 3591-3609.

(44) Dash, M.; Scemama, A., private communication.2018

(45) Boulanger, P.; Jacquemin, D.; Duchemin, I.; Blase, X. Fast and Accurate Electronic Excitations in Cyanines with the Many-Body Bethe-Slapter Approach. J. Chem. Theory Comput. 2014, 10, 12121218.

(46) Frisch, M. J.; Trucks, G. W.; Schlegel, H. B.; Scuseria, G. E.; Robb, M. A.; Cheeseman, J. R.; Scalmani, G.; Barone, V.; Petersson, G. A.; Nakatsuji, H.; Li, X.; Caricato, M.; Marenich, A.; Bloino, J.; Janesko, B. G.; Gomperts, R.; Mennucci, B.; Hratchian, H. P.; Ortiz, J. V.; Izmaylov, A. F.; Sonnenberg, J. L.; Williams-Young, D.; Ding, F.; Lipparini, F.; Egidi, F.; Goings, J.; Peng, B.; Petrone, A.; Henderson, T.; Ranasinghe, D.; Zakrzewski, V. G.; Gao, J.; Rega, N.; Zheng, G.; Liang, W.; Hada, M.; Ehara, M.; Toyota, K.; Fukuda, R.; Hasegawa, J.; Ishida, M.; Nakajima, T.; Honda, Y.; Kitao, O.; Nakai, H.; Vreven, T.;
Throssell, K.; Montgomery, J. A.; Peralta, J. J. E.; Ogliaro, F.; Bearpark, M.; Heyd, J. J.; Brothers, E.; Kudin, K. N.; Staroverov, V. N.; Keith, T.; Kobayashi, R.; Normand, J.; Raghavachari, K.; Rendell, A.; Burant, J. C.; Iyengar, S. S.; Tomasi, J.; Cossi, M.; Millam, J. M.; Klene, M.; Adamo, C.; Cammi, R.; Ochterski, J. W.; Martin, R. L.; Morokuma, K.; Farkas, O.; Foresman, J. B.; Fox, D. J. Gaussian09, Revision A.02; Gaussian, Inc.: Wallingford CT, 2016.

(47) Parrish, R. M.; Burns, L. A.; Smith, D. G. A.; Simmonett, A. C.; DePrince, A. E.; Hohenstein, E. G.; Bozkaya, U.; Sokolov, A. Y.; Di Remigio, R.; Richard, R. M.; Gonthier, J. F.; James, A. M.; McAlexander, H. R.; Kumar, A.; Saitow, M.; Wang, X.; Pritchard, B. P.; Verma, P.; Schaefer, H. F.; Patkowski, K.; King, R. A.; Valeev, E. F.; Evangelista, F. A.; Turney, J. M.; Crawford, T. D.; Sherrill, C. D. Psi4 1.1: An Open-Source Electronic Structure Program Emphasizing Automation, Advanced Libraries, and Interoperability. J. Chem. Theory Comput. 2017, 13, 3185-3197.

(48) Garniron, Y.; Scemama, A.; Giner, E.; Caffarel, M.; Loos, P.-F. Selected configuration interaction dressed by perturbation. J. Chem. Phys. 2018, 149, 064103. 